\author{
A. Ashyralyev ${ }^{1-3}$, F. Emharab ${ }^{4,5}$ \\ ${ }^{1}$ Near East University, Nicosia, Turkey; \\ ${ }^{2}$ Peoples' Friendship University of Russia, Moscow, Russia; \\ ${ }^{3}$ Institute of Mathematics and Mathematical Modeling, Almaty, Kazakhstan; \\ ${ }^{4}$ Near East University, Nicosia, Turkey; \\ ${ }^{5}$ Omar Al-Mukhtar University, El-Beida, Turkey \\ (E-mail: allaberen.ashyralyev@neu.edu.tr)
}

\title{
Identification hyperbolic problems with the Neumann boundary condition
}

\begin{abstract}
In the present study, an identification problem with the Neumann boundary condition for a one-dimensional hyperbolic equation is investigated. Stability estimates for the solution of the identification problem are established. Furthermore, a first order of accuracy difference scheme for the numerical solution of the identification problems for hyperbolic equations with the Neumann boundary condition is presented. Stability estimates for the solution of the difference scheme are established. This difference scheme is tested on an example and some numerical results are presented.
\end{abstract}

Keywords: source identification problem, hyperbolic differential equations, difference schemes.

\section{Introduction}

Identification problems take an important place in applied sciences and engineering, and have been studied by many authors (see, e.g., [1-4] and the references given therein). The theory and applications of source identification problems for partial differential equations have been given in various papers (see, e.g., [3, 5-8] and the references given therein).

The well-posedness of the unknown source identification problem for parabolic and delay parabolic equations have been well-investigated (see, e.g., [9-14], and the references given therein).

The solvability of the inverse problems in various formulations with various overdetermination conditions for telegraph and hyperbolic equations were studied in many works (see, e.g., [15-18] and the references given therein). Some new representations were given for the solutions and coefficients of the equations of mathematical physics in [5, 19-23]. They gave such formulas for evolution equations of first and second-order in time, in particular for parabolic and hyperbolic equations in the linear and nonlinear cases.

In this study, we consider the time-dependent source identification problem for a one-dimensional hyperbolic equation with the Neumann boundary condition

$$
\left\{\begin{array}{l}
\frac{\partial^{2} u(t, x)}{\partial t^{2}}-\frac{\partial}{\partial x}\left(a(x) \frac{\partial u(t, x)}{\partial x}\right)=p(t) q(x)+f(t, x), x \in(0, l), t \in(0, T), \\
u(0, x)=\varphi(x), u_{t}(0, x)=\psi(x), x \in[0, l] \\
u_{x}(t, 0)=u_{x}(t, l)=0, \int_{0}^{l} u(t, x) d x=\zeta(t), t \in[0, T]
\end{array}\right.
$$

where $u(t, x)$ and $p(t)$ are unknown functions, $a(x) \geq a>0, f(t, x), \zeta(t), \varphi(x)$ and $\psi(x)$ are sufficiently smooth functions and $q(x)$ is a sufficiently smooth function assuming $q^{\prime}(0)=q^{\prime}(l)=0$ and $\int_{0}^{l} q(x) d x \neq 0$.

Our interest in this study is to investigate the stability of differential and difference identification problems. The stability estimate for the solution of problem (1) is established. A first order of accuracy difference scheme for the numerical solution of identification hyperbolic problems with the Neumann boundary condition (1) is presented. The theoretical statements for solution of this difference scheme are supported by result of the numerical experiments. 
Stability of differential problem (1)

To formulate our results, we introduce the Banach space $C(H)=C([0, T], H)$ of all abstract continuous functions $\phi(t)$ defined on $[0, T]$ with values in $H$ equipped with the norm

$$
\|\phi\|_{C(H)}=\max _{0 \leqslant t \leqslant T}\|\phi(t)\|_{H} .
$$

Let $L_{2}[0, l]$ be a space of all square integrable functions $\gamma(x)$ defined on $[0, l]$ equipped with the norm

$$
\|\gamma\|_{L_{2}[0, l]}=\left(\int_{0}^{l}|\gamma(x)|^{2} d x\right)^{\frac{1}{2}},
$$

and let $W_{2}^{1}[0, l], W_{2}^{2}[0, l]$ be Sobolev spaces with norms

$$
\begin{aligned}
\|\gamma\|_{W_{2}^{1}[0, l]} & =\left(\int_{0}^{l}\left[\gamma^{2}(x)+\gamma_{x}^{2}(x)\right] d x\right)^{\frac{1}{2}} \\
\|\gamma\|_{W_{2}^{2}[0, l]} & =\left(\int_{0}^{l}\left[\gamma^{2}(x)+\gamma_{x x}^{2}(x)\right] d x\right)^{\frac{1}{2}},
\end{aligned}
$$

respectively. We introduce the positive definite self-adjoint operator $A$ defined by the formula

$$
A u=-\frac{d}{d x}\left(a(x) \frac{d u(x)}{d x}\right)
$$

with the domain

$$
D(A)=\left\{u: u, u^{\prime \prime} \in L_{2}[0, l], u^{\prime}(0)=u^{\prime}(l)=0\right\} .
$$

Throughout the present paper, $M$ denotes positive constants, which may differ in time and thus is not a subject of precision. However, we will use the notation $M(\alpha, \beta, \gamma, \ldots)$ to stress the fact that the constant depends only on $\alpha, \beta, \gamma, \ldots$

We have the following theorem on the stability of problem (1).

Theorem 1. Assume that $\varphi \in W_{2}^{2}[0, l], \psi \in W_{2}^{1}[0, l]$ and $f(t, x)$ is a continuously differentiable function in $t$ and square integrable in $x, \zeta(t)$ is a twice continuously differentiable function. Suppose that $q(x)$ is a sufficiently smooth function assuming $q^{\prime}(0)=q^{\prime}(l)=0$ and $\int_{0}^{l} q(x) d x \neq 0$. Then for the solution of problem (1) the following stability estimates hold

$$
\begin{gathered}
\left\|\frac{\partial^{2} u}{\partial t^{2}}\right\|_{C\left(L_{2}[0, l]\right)}+\|u\|_{C\left(W_{2}^{2}[0, l]\right)} \leqslant M_{1}(q)\left[\|\varphi\|_{W_{2}^{2}[0, l]}+\|\psi\|_{W_{2}^{1}[0, l]}+\right. \\
\left.+\|f(0, \cdot)\|_{L_{2}[0, l]}+\left\|\frac{\partial f}{\partial t}\right\|_{C\left(L_{2}[0, l]\right)}+\left\|\zeta^{\prime \prime}\right\|_{C[0, T]}\right] \\
\|p\|_{C[0, T]} \leqslant M_{2}(q)\left[\|\varphi\|_{W_{2}^{2}[0, l]}+\|\psi\|_{W_{2}^{1}[0, l]}+\left\|\zeta^{\prime \prime}\right\|_{C[0, T]}+\right. \\
\left.+\|f(0, \cdot)\|_{L_{2}[0, l]}+\left\|\frac{\partial f}{\partial t}\right\|_{C\left(L_{2}[0, l]\right)}\right]
\end{gathered}
$$

Proof. We will use the following substitution

$$
u(t, x)=w(t, x)+\eta(t) q(x),
$$

where $\eta(t)$ is the function defined by the formula

$$
\eta(t)=\int_{0}^{t}(t-s) p(s) d s, \eta(0)=\eta^{\prime}(0)=0
$$


It is easy to see that $w(t, x)$ is the solution of problem

$$
\left\{\begin{array}{l}
\frac{\partial^{2} w(t, x)}{\partial t^{2}}-\frac{\partial}{\partial x}\left(a(x) \frac{\partial w(t, x)}{\partial x}\right)=f(t, x)+\eta(t)\left[\frac{d}{d x}\left(a(x) q^{\prime}(x)\right)\right] \\
x \in(0, l), t \in(0, T) \\
w(0, x)=\varphi(x), w_{t}(0, x)=\psi(x), x \in[0, l] \\
u_{x}(t, 0)=u_{x}(t, l)=0,, t \in[0, T]
\end{array}\right.
$$

Applying the integral overdetermined condition $\int_{0}^{l} u(t, x) d x=\zeta(t)$ and substitution (5), we get

$$
\eta(t)=\frac{\zeta(t)-\int_{0}^{l} w(t, x) d x}{\int_{0}^{l} q(x) d x} .
$$

From that and $p(t)=\eta^{\prime \prime}(t)$ it follows

$$
p(t)=\frac{\zeta^{\prime \prime}(t)-\int_{0}^{l} \frac{\partial^{2}}{\partial t^{2}} w(t, x) d x}{\int_{0}^{l} q(x) d x} .
$$

Applying $\int_{0}^{l} q(x) d x \neq 0$, we get the estimate

$$
|p(t)| \leq M_{3}(q)\left[\left|\zeta^{\prime \prime}(t)\right|+\left\|\frac{\partial^{2} w(t, .)}{\partial t^{2}}\right\|_{L_{2}[0, l]}\right]
$$

for all $t \in[0, T]$. From that it follows

$$
\|p\|_{C[0, T]} \leqslant M_{3}(q)\left[\left\|\zeta^{\prime \prime}\right\|_{C[0, T]}+\left\|\frac{\partial^{2} w}{\partial t^{2}}\right\|_{C\left(L_{2}[0, l]\right)}\right] .
$$

Now, using substitution (5), we get

$$
\frac{\partial^{2} u(t, x)}{\partial t^{2}}=\frac{\partial^{2} w(t, x)}{\partial t^{2}}+p(t) q(x) .
$$

Applying the triangle inequality, we obtain

$$
\left\|\frac{\partial^{2} u}{\partial t^{2}}\right\|_{C\left(L_{2}[0, l]\right)} \leqslant\left\|\frac{\partial^{2} w}{\partial t^{2}}\right\|_{C\left(L_{2}[0, l]\right)}+\|p\|_{C[0, T]}\|q\|_{L_{2}[0, l]} .
$$

Therefore, the proof of estimates (3) and (4) is based on equation (1), the triangle inequality, estimates (9), (10) and on the following stability estimate

$$
\begin{gathered}
\left\|\frac{\partial^{2} w}{\partial t^{2}}\right\|_{C\left(L_{2}[0, l]\right)} \leqslant M_{4}(q, a)\left[\|\varphi\|_{W_{2}^{2}[0, l]}+\|\psi\|_{W_{2}^{1}[0, l]}+\right. \\
\left.+\|f(0, \cdot)\|_{L_{2}[0, l]}+\left\|\frac{\partial f}{\partial t}\right\|_{C\left(L_{2}[0, l]\right)}+\left\|\zeta^{\prime \prime}\right\|_{C[0, T]}\right]
\end{gathered}
$$

for the solution of problem (7). It was proved in [16] for the identification hyperbolic problem with the Dirichlet boundary condition. The proof of (11) is carried out according to the same approach. This completes the proof of Theorem 1. 


\section{Stability of the difference scheme}

To formulate our results on difference problem, we introduce the Banach space $C_{\tau}(H)=C\left([0, T]_{\tau}, H\right)$ of all abstract grid functions $\phi^{\tau}=\left\{\phi\left(t_{k}\right)\right\}_{k=0}^{N}$ defined on

$$
[0, T]_{\tau}=\left\{t_{k}=k \tau, 0 \leqslant k \leqslant N, N \tau=T\right\},
$$

with values in $H$ equipped with the norm

$$
\left\|\phi^{\tau}\right\|_{C_{\tau}(H)}=\max _{0 \leqslant k \leqslant N}\left\|\phi\left(t_{k}\right)\right\|_{H} .
$$

Moreover, $L_{2 h}=L_{2}[0, l]_{h}$ is the Hilbert space of all grid functions $\gamma^{h}(x)=\left\{\gamma_{n}\right\}_{n=0}^{M}$ defined on

$$
[0, l]_{h}=\left\{x_{n}=n h, 0 \leqslant n \leqslant M, M h=l\right\},
$$

equipped with the norm

$$
\left\|\gamma^{h}\right\|_{L_{2 h}}=\left\{\sum_{i=0}^{M}\left|\gamma_{i}\right|^{2} h\right\}^{\frac{1}{2}},
$$

and $W_{2 h}^{1}=W_{2}^{1}[0, l]_{h}, W_{2 h}^{2}=W_{2}^{2}[0, l]_{h}$ are the discrete analogues of Sobolev spaces of all grid functions $\gamma^{h}(x)=\left\{\gamma_{n}\right\}_{n=0}^{M}$ defined on $[0, l]_{h}$ with norms

$$
\begin{gathered}
\left\|\gamma^{h}\right\|_{W_{2 h}^{1}}=\left\{\sum_{i=0}^{M}\left|\gamma_{i}\right|^{2} h+\sum_{i=1}^{M}\left|\frac{\gamma_{i}-\gamma_{i-1}}{h}\right|^{2} h\right\}^{\frac{1}{2}} ; \\
\left\|\gamma^{h}\right\|_{W_{2 h}^{2}}=\left\{\sum_{i=0}^{M}\left|\gamma_{i}\right|^{2} h+\sum_{i=1}^{M-1}\left|\frac{\gamma_{i+1}-2 \gamma_{i}+\gamma_{i-1}}{h^{2}}\right|^{2} h\right\}^{\frac{1}{2}},
\end{gathered}
$$

respectively. For the differential operator $A$ defined by (2), we introduce the self-adjoint positive definite difference operator $A_{h}$ defined by the formula

$$
A_{h} \varphi^{h}(x)=\left\{-\frac{1}{h}\left(a\left(x_{n+1}\right) \frac{\varphi_{n+1}-\varphi_{n}}{h}-a\left(x_{n}\right) \frac{\varphi_{n}-\varphi_{n-1}}{h}\right)\right\}_{n=1}^{M-1}
$$

acting in the space of grid functions $\varphi^{h}(x)=\left\{\varphi_{n}\right\}_{n=0}^{M}$ defined on $[0, l]_{h}$ satisfying the conditions $\varphi_{1}-\varphi_{0}=\varphi_{M}-\varphi_{M-1}=0$. scheme

For the numerical solution $\left\{\left\{u_{n}^{k}\right\}_{k=0}^{N}\right\}_{n=0}^{M}$ of problem (1), we consider the first order of accuracy difference

$$
\left\{\begin{array}{l}
\frac{u_{n}^{k+1}-2 u_{n}^{k}+u_{n}^{k-1}}{\tau^{2}}-\left(a\left(x_{n+1}\right) \frac{u_{n+1}^{k+1}-u_{n}^{k+1}}{h^{2}}-a\left(x_{n}\right) \frac{u_{n}^{k+1}-u_{n-1}^{k+1}}{h^{2}}\right)=p_{k} q\left(x_{n}\right)+f\left(t_{k}, x_{n}\right) ; \\
t_{k}=k \tau, x_{n}=n h, 1 \leqslant k \leqslant N-1,1 \leqslant n \leqslant M-1, N \tau=T ; \\
u_{n}^{0}=\varphi\left(x_{n}\right), \frac{u_{n}^{1}-u_{n}^{0}}{\tau}=\psi\left(x_{n}\right), 0 \leqslant n \leqslant M, M h=l ; \\
u_{1}^{k+1}-u_{0}^{k+1}=u_{M}^{k+1}-u_{M-1}^{k+1}=0 ; \\
\sum_{i=1}^{M-1} u_{i}^{k+1} h=\zeta\left(t_{k+1}\right),-1 \leqslant k \leqslant N-1 .
\end{array}\right.
$$

Here, it is assumed that $q_{1}-q_{0}=q_{M}-q_{M-1}=0$ and $\sum_{i=1}^{M-1} q_{i} \neq 0$. We have the following theorem on the stability of difference scheme (13). 
Theorem 2. For the solution of difference scheme (13), the following stability estimates hold

$$
\begin{gathered}
\left\|\left\{\frac{u_{k+1}^{h}-2 u_{k}^{h}+u_{k-1}^{h}}{\tau^{2}}\right\}_{k=1}^{N-1}\right\|_{C_{\tau}\left(L_{2 h}\right)}+\left\|\left\{u_{k+1}^{h}\right\}_{k=1}^{N-1}\right\|_{C_{\tau}\left(W_{2 h}^{2}\right)} \leqslant \\
\leqslant M_{5}(q)\left[\left\|\varphi^{h}\right\|_{W_{2 h}^{2}}+\left\|\psi^{h}\right\|_{W_{2 h}^{1}}+\left\|f_{1}^{h}\right\|_{L_{2 h}}+\left\|\left\{\frac{f_{k}^{h}-f_{k-1}^{h}}{\tau}\right\}_{k=2}^{N-1}\right\|_{C_{\tau}\left(L_{2 h}\right)}+\right. \\
\left.+\left\|\left\{\frac{\zeta_{k+1}-2 \zeta_{k}+\zeta_{k-1}}{\tau^{2}}\right\}_{k=1}^{N-1}\right\| \|_{C[0, T]_{\tau}}\right], \\
\left.+\left\|\left\{\frac{\left.p_{k}\right\}_{k=1}^{N-1} \|_{C[0, T]_{\tau}} \leqslant M_{6}(q)\left[\left\|\varphi^{h}\right\|_{W_{2 h}^{2}}+\left\|\psi^{h}\right\|_{W_{2 h}^{2}}+\left\|f_{1}^{h}\right\|_{L_{2 h}}+\right.}{\tau}+\|\left\{\frac{\zeta_{k+1}^{h}-2 \zeta_{k-1}^{h}+\zeta_{k-1}}{\tau^{2}}\right\}_{k=2}^{N-1}\right\}_{k=1}^{N-1}\right\|_{C[0, T]_{\tau}}\right] .
\end{gathered}
$$

Here and throughout this subsection $f_{k}^{h}(x)=\left\{f\left(t_{k}, x_{n}\right)\right\}_{n=0}^{M}, 1 \leq k \leq N-1$.

Proof. We will use the following substitution

$$
u_{n}^{k}=w_{n}^{k}+\eta_{k} q_{n}
$$

where

$$
q_{n}=q\left(x_{n}\right)
$$

and

$$
\eta_{k+1}=\sum_{i=1}^{k}(k+1-i) p_{i} \tau^{2}, 1 \leqslant k \leqslant N-1, \eta_{0}=\eta_{1}=0 .
$$

It is easy to see that $\left\{\left\{w_{n}^{k}\right\}_{k=0}^{N}\right\}_{n=0}^{M}$ is the solution of difference problem

$$
\left\{\begin{array}{l}
\frac{w_{n}^{k+1}-2 w_{n}^{k}+w_{n}^{k-1}}{\tau^{2}}-\frac{1}{h}\left(a\left(x_{n+1}\right) \frac{w_{n+1}^{k+1}-w_{n}^{k+1}}{h}-a\left(x_{n}\right) \frac{w_{n}^{k+1}-w_{n-1}^{k+1}}{h}\right)= \\
=f\left(t_{k}, x_{n}\right)+\frac{1}{h}\left[a\left(x_{n+1}\right) \frac{q_{n+1}-q_{n}}{h}-a\left(x_{n}\right) \frac{q_{n}-q_{n-1}}{h}\right] \eta_{k+1} \\
1 \leqslant k \leqslant N-1,1 \leqslant n \leqslant M-1 ; \\
w_{n}^{0}=\varphi\left(x_{n}\right), \frac{w_{n}^{1}-w_{n}^{0}}{\tau}=\psi\left(x_{n}\right), 0 \leqslant n \leqslant M \\
u_{1}^{k+1}-u_{0}^{k+1}=u_{M}^{k+1}-u_{M-1}^{k+1}=0,-1 \leqslant k \leqslant N-1 .
\end{array}\right.
$$

Applying the overdetermined condition $\sum_{i=1}^{M-1} u_{i}^{k+1} h=\zeta\left(t_{k+1}\right)$ and substitution (16), one can obtain that

$$
\eta_{k+1}=\frac{\zeta_{k+1}-\sum_{i=1}^{M-1} w_{i}^{k+1} h}{\sum_{i=1}^{M-1} q_{i} h} .
$$

Then, using formulas $p_{k}=\frac{\eta_{k+1}-2 \eta_{k}+\eta_{k-1}}{\tau^{2}}$ and (19), we get

$$
p_{k}=\frac{\zeta_{k+1}-2 \zeta_{k}+\zeta_{k-1}-\sum_{i=1}^{M-1}\left(w_{i}^{k+1}-2 w_{i}^{k}+w_{i}^{k-1}\right) h}{\tau^{2} \sum_{i=1}^{M-1} q_{i} h},
$$




$$
\left|p_{k}\right| \leqslant M_{7}(q)\left[\left|\frac{\zeta_{k+1}-2 \zeta_{k}+\zeta_{k-1}}{\tau^{2}}\right|+\left\|\frac{w_{k+1}^{h}-2 w_{k}^{h}+w_{k-1}^{h}}{\tau^{2}}\right\|_{L_{2 h}}\right]
$$

for all $1 \leqslant k \leqslant N-1$. From that it follows

$$
\begin{gathered}
\left\|\left\{p_{k}\right\}_{k=1}^{N-1}\right\|_{C[0, T]_{\tau}} \leqslant M_{7}(q)\left[\left\|\left\{\frac{\zeta_{k+1}-2 \zeta_{k}+\zeta_{k-1}}{\tau^{2}}\right\}_{k=1}^{N-1}\right\|_{C[0, T]_{\tau}}+\right. \\
\left.+\left\|\left\{\frac{w_{k+1}^{h}-2 w_{k}^{h}+w_{k-1}^{h}}{\tau^{2}}\right\}_{k=1}^{N-1}\right\|_{C_{\tau}\left(L_{2 h}\right)}\right] .
\end{gathered}
$$

Now, using substitution (16), we get

$$
\frac{u_{n}^{k+1}-2 u_{n}^{k}+u_{n}^{k-1}}{\tau^{2}}=\frac{w_{n}^{k+1}-2 w_{n}^{k}+w_{n}^{k-1}}{\tau^{2}}+p_{k} q\left(x_{n}\right) .
$$

Applying the triangle inequality, we obtain

$$
\begin{aligned}
& \left\|\left\{\frac{u_{k+1}^{h}-2 u_{k}^{h}+u_{k-1}^{h}}{\tau^{2}}\right\}_{k=1}^{N-1}\right\|_{C_{\tau}\left(L_{2 h}\right)} \leqslant \\
& \leqslant\left\|\left\{\frac{w_{k+1}^{h}-2 w_{k}^{h}+w_{k-1}^{h}}{\tau^{2}}\right\}_{k=1}^{N-1}\right\|_{C_{\tau}\left(L_{2 h}\right)}+ \\
& +\left\|\left\{p_{k}\right\}_{k=1}^{N-1}\right\|_{C[0, T]_{\tau}}\left\|\left\{q\left(x_{n}\right)\right\}_{n=0}^{M}\right\|_{L_{2 h}} .
\end{aligned}
$$

Therefore, the proof of estimates (14) and (15) are based on equation (13), the triangle inequality, estimates (21), (22) and on the following stability estimate

$$
\begin{gathered}
\left\|\left\{\frac{w_{k+1}^{h}-2 w_{k}^{h}+w_{k-1}^{h}}{\tau^{2}}\right\}_{k=1}^{N-1}\right\|_{C_{\tau}\left(L_{2 h}\right)} \leqslant M_{8}(q) \times \\
\times\left[\left\|\varphi^{h}\right\|_{W_{2 h}^{2}}+\left\|\psi^{h}\right\|_{W_{2 h}^{1}}+\left\|f_{1}^{h}\right\|_{L_{2 h}}+\left\|\left\{\frac{f_{k}^{h}-f_{k-1}^{h}}{\tau}\right\}_{k=2}^{N-1}\right\|_{C_{\tau}\left(L_{2 h}\right)}+\right. \\
\left.+\left\|\left\{\frac{\zeta_{k+1}-2 \zeta_{k}+\zeta_{k-1}}{\tau^{2}}\right\}_{k=1}^{N-1}\right\|_{C[0, T]_{\tau}}\right]
\end{gathered}
$$

for the solution of difference problem (18). It was proved in ([9]) for the identification hyperbolic problem with the Dirichlet boundary condition. The proof of (23) is carried out according to the same approach. This completes the proof of Theorem 2.

\section{Numerical Experiments}

In this section, we study the numerical solution of the identification problem

$$
\left\{\begin{array}{l}
\frac{\partial^{2} u(t, x)}{\partial t^{2}}-\frac{\partial^{2} u(t, x)}{\partial x^{2}}=p(t)(1+\cos x)+e^{-t} \cos x, x \in(0, \pi), t \in(0,1) \\
u(0, x)=1+\cos x, u_{t}(0, x)=-(1+\cos x), x \in[0, \pi] \\
u_{x}(t, 0)=u_{x}(t, \pi)=0, t \in[0,1] \\
\int_{0}^{\pi} u(t, x) d x=\pi e^{-t}, t \in[0,1]
\end{array}\right.
$$

for a hyperbolic differential equation. The exact solution pair of this problem is $(u(t, x), p(t))=$ $=\left(e^{-t}(1+\cos x), e^{-t}\right)$. 
For the numerical solution of problem (24), we present the following first order of accuracy difference scheme for the approximate solution for the problem (24)

$$
\left\{\begin{array}{l}
\frac{u_{n}^{k+1}-2 u_{n}^{k}+u_{n}^{k-1}}{\tau^{2}}-\frac{u_{n+1}^{k+1}-2 u_{n}^{k+1}+u_{n-1}^{k+1}}{h^{2}}=p_{k}\left(1+\cos x_{n}\right)+e^{-t_{k+1}} \cos x_{n} \\
t_{k}=k \tau, x_{n}=n h, 1 \leqslant k \leqslant N-1,1 \leqslant n \leqslant M-1 \\
u_{n}^{0}=1+\cos x_{n}, \frac{u_{n}^{1}-u_{n}^{0}}{\tau}=-\left(1+\cos x_{n}\right), 0 \leqslant n \leqslant M, M h=\pi, N \tau=1 \\
u_{0}^{k+1}-u_{1}^{k+1}=u_{M}^{k+1}-u_{M-1}^{k+1}=0 \\
\sum_{i=1}^{M-1} u_{i}^{k+1} h=\pi e^{-t_{k+1}},-1 \leqslant k \leqslant N-1 .
\end{array}\right.
$$

Algorithm for obtaining the solution of identification problem (25) contains three stages. Actually, let us define

$$
u_{n}^{k}=w_{n}^{k}+\eta_{k}\left(1+\cos x_{n}\right), 0 \leq k \leq N, 0 \leq n \leq M,
$$

Applying difference scheme (25) and formula (26), we will obtain the formula

$$
\eta_{k+1}=\frac{\pi e^{-t_{k+1}}-\sum_{i=1}^{M-1} w_{i}^{k+1} h}{\sum_{i=1}^{M-1}\left(1+\cos x_{i}\right) h},-1 \leq k \leq N-1
$$

and the difference scheme

$$
\left\{\begin{array}{l}
\frac{w_{n}^{k+1}-2 w_{n}^{k}+w_{n}^{k-1}}{\tau^{2}}-\frac{w_{n+1}^{k+1}-2 w_{n}^{k+1}+w_{n-1}^{k+1}}{h^{2}}+\frac{2(\cos h-1)}{h^{2}} \cos x_{n} \frac{\sum_{i=1}^{M-1} w_{i}^{k+1} h}{\sum_{i=1}^{M-1}\left(1+\cos x_{i}\right) h}= \\
=\left[1+\frac{2 \pi(\cos h-1)}{h^{2} \sum_{i=1}^{M-1}\left(1+\cos x_{i}\right) h}\right] e^{-t_{k+1} \cos x_{n}, 1 \leqslant k \leqslant N-1,1 \leqslant n \leqslant M-1} \\
w_{n}^{0}=1+\cos x_{n}, \frac{w_{n}^{1}-w_{n}^{0}}{\tau}=-\left(1+\cos x_{n}\right), 0 \leqslant n \leqslant M \\
w_{0}^{k+1}-w_{1}^{k+1}=w_{M}^{k+1}-w_{M-1}^{k+1}=0,-1 \leqslant k \leqslant N-1 .
\end{array}\right.
$$

In the first stage, we find numerical solution $\left\{\left\{w_{n}^{k}\right\}_{k=0}^{N}\right\}_{n=0}^{M}$ of corresponding first order of accuracy auxiliary difference scheme (28). For obtaining the solution of difference scheme (28), we will write it in the matrix form as

$$
\left\{\begin{array}{l}
A w^{k+1}+B w^{k}+C w^{k-1}=\varphi^{k}, 1 \leqslant k \leqslant N-1 ; \\
w^{0}=\left\{1+\cos x_{n}\right\}_{n=0}^{M}, w^{1}=\left\{(1-\tau)\left(1+\cos x_{n}\right)\right\}_{n=0}^{M},
\end{array}\right.
$$

where $A, B, C$ are $(M+1) \times(M+1)$ square matrices, $w^{s}, s=k, k \pm 1, f^{k}$ are $(M+1) \times 1$ column matrices and

$$
\begin{gathered}
A=\left[\begin{array}{cccccccc}
1 & -1 & 0 & \cdot & 0 & 0 & -1 \\
b & a+c_{1} & b+c_{1} & \cdot & c_{1} & c_{1} & 0 \\
0 & b+c_{2} & a+c_{2} & \cdot & c_{2} & c_{2} & 0 \\
\cdot & \cdot & \cdot & \cdot & \cdot & \cdot & \cdot \\
0 & c_{M-2} & c_{M-2} & \cdot & a+c_{M-2} & b+c_{M-2} & 0 \\
0 & c_{M-1} & c_{M-1} & \cdot & b+c_{M-1} & a+c_{M-1} & b \\
0 & 0 & 0 & \cdot & 0
\end{array}\right]_{(M+1) \times(M+1)} \\
B=\left[\begin{array}{ccccccc}
0 & 0 & 0 & \cdot & 0 & 0 & 0 \\
0 & e & 0 & \cdot & 0 & 0 & 0 \\
0 & 0 & e & \cdot & 0 & 0 & 0 \\
\cdot & \cdot & \cdot & \cdot & \cdot & \cdot & \cdot \\
0 & 0 & 0 & \cdot & e & 0 & 0 \\
0 & 0 & 0 & \cdot & 0 & e & 0 \\
0 & 0 & 0 & \cdot & 0 & 0 & 0
\end{array}\right]_{(M+1) \times(M+1)} \quad\left[\begin{array}{ccccccc}
0 & 0 & 0 & \cdot & 0 & 0 & 0 \\
0 & g & 0 & \cdot & 0 & 0 & 0 \\
0 & 0 & g & \cdot & 0 & 0 & 0 \\
\cdot & \cdot & \cdot & \cdot & \cdot & \cdot & \cdot \\
0 & 0 & 0 & \cdot & g & 0 & 0 \\
0 & 0 & 0 & \cdot & 0 & g & 0 \\
0 & 0 & 0 & \cdot & 0 & 0 & 0
\end{array}\right]_{(M+1) \times(M+1)}
\end{gathered}
$$




$$
\varphi^{k}=\left[\begin{array}{c}
0 \\
\varphi_{1}^{k} \\
\cdot \\
\varphi_{M-1}^{k} \\
0
\end{array}\right]_{(M+1) \times 1} \quad w^{s}=\left[\begin{array}{c}
0 \\
w_{1}^{s} \\
\cdot \\
w_{M-1}^{s} \\
0
\end{array}\right]_{(M+1) \times 1} \quad, \text { for } s=k, k \pm 1
$$

Here,

$$
\begin{gathered}
a=\frac{1}{\tau^{2}}+\frac{2}{h^{2}}, b=-\frac{1}{h^{2}}, e=-\frac{2}{\tau^{2}}, g=\frac{1}{\tau^{2}} ; \\
d=\sum_{i=1}^{M-1}\left(1+\cos x_{i}\right) h, c_{n}=\frac{2(\cos h-1)}{h d} \cos x_{n}, 1 \leqslant n \leqslant M-1, \\
\varphi_{n}^{k}=\left[1+\frac{2 \pi(\cos h-1)}{h^{2} \sum_{i=1}^{M-1}\left(1+\cos x_{i} h\right)}\right] e^{-t_{k+1}} \cos x_{n}, 1 \leqslant k \leqslant N-1,1 \leqslant n \leqslant M-1 .
\end{gathered}
$$

So, we have the initial value problem for the second order difference equation (29) with respect to $k$ with matrix coefficients $A, B$ and $C$. Since $w^{0}$ and $w^{1}$ are given, we can obtain $\left\{\left\{w_{n}^{k}\right\}_{k=0}^{N}\right\}_{n=0}^{M}$ by (29).

Now, applying formula (17), we can obtain

$$
p_{k}=\frac{\eta_{k+1}-2 \eta_{k}+\eta_{k-1}}{\tau^{2}}, 1 \leq k \leq N-1 .
$$

In the second stage, we obtain $\left\{p_{k}\right\}_{k=1}^{N-1}$ by formulas (27) and (30). Finally, in the third stage, we obtain $\left\{\left\{u_{n}^{k}\right\}_{k=0}^{N}\right\}_{n=0}^{M}$ by formulas (26) and (27). The errors are computed by

$$
\begin{gathered}
E_{u}=\max _{0 \leqslant k \leqslant N}\left(\sum_{n=0}^{M}\left|u\left(t_{k}, x_{n}\right)-u_{n}^{k}\right|^{2} h\right)^{\frac{1}{2}} ; \\
E_{p}=\max _{1 \leqslant k \leqslant N-1}\left|p\left(t_{k}\right)-p_{k}\right|,
\end{gathered}
$$

where $u(t, x), p(t)$ represent the exact solution, $u_{n}^{k}$ represent the numerical solutions at $\left(t_{k}, x_{n}\right)$ and $p_{k}$ represent the numerical solutions at $t_{k}$. The numerical results are given in the following Table.

Table

\section{Error analysis}

\begin{tabular}{|c|c|c|c|c|}
\hline Error & $N=M=20$ & $N=M=40$ & $N=M=80$ & $\mathrm{~N}=\mathrm{M}=160$ \\
\hline$E_{u}$ & 0.0501 & 0.0250 & 0.0124 & 0.0062 \\
\hline$E_{p}$ & 0.0472 & 0.0244 & 0.0124 & 0.0063 \\
\hline
\end{tabular}

As it is seen in Table, we get some numerical results. If $N$ and $M$ are doubled, the value of errors decrease by a factor of approximately $1 / 2$ for first order difference scheme (25).

\section{Acknowledgement}

The publication has been prepared with the support of the «RUDN University Program 5-100» and published under target program BR05236656 of the Science Committee of the Ministry of Education and Science of the Republic of Kazakhstan. We would like to thank the referees for their helpful suggestions to the improvement of our paper. 


\title{
References
}

1 Belov, Yu.Ya. (2002). Inverse problems for partial differential equations (Inverse and Ill-Posed Problems Series, VSP).

2 Isakov, V. (1998). Inverse problems for partial differential equations, Applied Mathematical Sciences (Springer, New York).

3 Kozhanov, A.I. (1997). Journal of Inverse and III-posed Problems, 5, 4, 337-352.

4 Prilepko, A.I., Orlovsky, D.G., \& Vasin, I.A. (2000). Methods for Solving Inverse Problems in Mathematical Physics. New York: Marcel Dekker.

5 Anikonov, Yu.E. (1996). Siberian Mathematical Journal, 37, 3, 483-491.

6 Ashyralyev, A., (2014). \& Ashyralyyev, Ch. Nonlinear Analysis: Modelling and Control), 19, 3, 350-366.

7 Ashyralyyev, Ch. (2014). Boundary Value Problem, 2014, 5, 1-23.

8 Orlovskii, D.G. (2008). Differential Equations, 44, 1, 124-134.

9 Ashyralyev, A. (2011). Ukrainian Mathematical Journal, 62, 9, 1397-1408.

10 Ashyralyev, A., \& Agirseven, D. (2014). Nonlinear Analysis: Modelling and Control, 19, 3, 335-349.

11 Blasio, G.Di., \& Lorenzi, A. (2007). Journal of Inverse and III-posed Problems, 15, 7, 709-734.

12 Ivanchov, N.I. (1995). Ukrainian Mathematical Journal, 47, 10, 1647-1652.

13 Kostin, A.B. (2013). Sbornik: Mathematics, 204, 10, 1391-1434.

14 Samarskii, A.A., \& Vabishchevich, P.N. (2007). Numerical Methods for Solving Inverse Problems of Mathematical Physics. New York: Berlin. Inverse and Ill-Posed Problems Series, Walter de Gruyter.

15 Ashyralyev, A., \& Celik, F. (2016). Series Springer Proceedings in Mathematics and Statistics, 39-50. Cham: Publishing.

16 Ashyralyev, A., \& Emharab, F. (2017). AIP Conference Proceedings, 1880,050001, doi:10.1063/1.5000617.

17 Kozhanov, A.I., \& Safiullova, R.R. (2010). Journal of Inverse and III-posed Problems, 18, 1, 1-24.

18 Kozhanov, A.I., \& Telesheva, L.A. (2017). Bulletin of the south Ural State University, Series: Mathematical Modelling, Programming and Computer Software, 10, 2, 24-37.

19 Anikonov, Yu.E. (1995). Journal of Inverse and III-posed Problems, 3, 4, 259-267.

20 Anikonov, Yu.E., \& Neshchadim, M.V. (2012). Journal of Applied and Industrial Mathematics, 6, 1, 6-11.

21 Anikonov, Yu.E., \& Neshchadim, M.V. (2013). Journal of Applied and Industrial Mathematics, 7, 1, $15-21$.

22 Ashyralyev, A., \& Sobolevskii, P.E. (2004). New Difference Schemes for Partial Differential Equations. Basel, Boston, Berlin: Operator Theory Advances and Applications, Birkhauser Verlag.

23 Kabanikhin, S.I., \& Krivorotko, O.I. (2015). Journal of Inverse and III-posed Problems, 23, 5, 519-527.

\author{
А. Ашыралыев, Ф. Эммараб
}

\section{Шеттік шарты Нейман түрінде болатын идентификациялық гиперболалық есептер}

\begin{abstract}
Мақала шеттік шарты Нейман түріндегі бір өлшемді гиперболалық түрдегі теңдеу үшін идентификациялау есебін зерттеуге арналған. Идентификациялау есебінің шешімі үшін орнықтылық бағалаулары алынған. Шеттік шарты Нейман түріндегі гиперболалық теңдеулер үшін идентификациялау есебін сандық шешу үшін дәлдігі бірінші ретті айырымдық схема ұсынылған. Айырымдық схеманың шешімі үшін орнықтылық бағалаулары келтірілген. Бұл айырымдық схема қарапайым есеп үшін тексеріліп, сандық есептеулер нәтижесі келтірілген.

Kiлm сөздер: көздерді идентификациялау есебі, гиперболалық дифференциалды теңдеу, айырымдық схемалары.
\end{abstract}




\section{А. Ашыралыев, Ф. Эммараб \\ Идентификационные гиперболические задачи с граничным условием Неймана}

В статье изучаена задача идентификации с граничным условием Неймана для одномерного гиперболического уравнения. Установлены оценки устойчивости решения задачи идентификации. Кроме того, представлена разностная схема первого порядка точности для численного решения задач идентификации для гиперболических уравнений с граничным условием Неймана. Установлены оценки устойчивости решения разностной схемы. Эта разностная схема проверена на примере и представлены некоторые численные результаты.

Ключевые слова: задача идентификации источника, гиперболические дифференциальные уравнения, разностные схемы. 